\title{
Stability of nonlinear Volterra equations
}

\author{
P. H. A. NGOC ${ }^{1 *}$ and L. T. HIEU ${ }^{2}$ \\ ${ }^{1}$ Department of Mathematics, Vietnam National University-HCMC, International University, Saigon, Vietnam \\ ${ }^{2}$ Department of Mathematics, Dong Thap University, Cao Lanh city, Dong Thap, Vietnam
}

\begin{abstract}
Using a novel approach, we present some new explicit criteria for global exponential stability of the zero solution of general nonlinear time-varying Volterra difference equations. Furthermore, an explicit stability bound for equations subject to nonlinear time-varying perturbations is given. Finally, the obtained results are used to study uniform attraction of equilibrium of discrete-time bidirectional associative memory (BAM) neural networks. Some illustrative examples are given.
\end{abstract}

Key words: global exponential stability, Volterra equations.

\section{Introduction}

Volterra difference equations have been widely used in the modeling of processes in continuous mechanics and biomechanics, problems of control and estimations and some schemes of numerical solutions of integral and integro-differential equations, see e.g. [2, 7, 12, 16-19].

Problems of stability of Volterra difference equations have attracted much attention from researchers, during the last twenty years, see e.g. [1, 4-13, 15, 21-23] and references therein. Many various methods have been used to investigate stability of Volterra difference equations such as Lyapunov functions, $\mathrm{Z}$ transform, comparison theorems, topological methods, fixed point theorems, etc. (see e.g. [7, 10, 15, 23]).

In particular, the first significant result on exponential stability of Volterra difference equations was achieved by Elaydi and Murakami, see [8]. Roughly speaking, Elaydi and Murakami showed that even the simplest Volterra difference equations (namely, linear time-invariant Volterra difference equations of convolution type) have "stronger" exponential stability than the uniform asymptotic stability. More precisely, a linear time-invariant Volterra difference equation of convolution type is exponentially stable if and only if it is uniformly asymptotically stable and its kernel exponentially decays, see [8]. For further and updated information on stability of linear time-invariant Volterra difference equations, we refer to the recent survey paper [10].

In general, problems of global exponential stability of timevarying Volterra difference equations are difficult, even when concerning linear time-varying Volterra equations. Most of existing results in the literature are derived by the method of Lyapunov functions and they are not easy to use. Some sufficient conditions for exponential stability of linear time-varying Volterra difference equations can be found in $[5,10,15]$. To the best of our knowledge, there are not many explicit criteria for

*e-mail: phangoc@hcmiu.edu.vn global exponential stability of nonlinear time-varying Volterra difference equations in the literature. In particular, several abstract criteria for exponential stability of some nonlinear Volterra difference equations can be found in $[5,15,22]$.

In this paper, we present a new approach to global exponential stability of nonlinear time-varying Volterra difference equations. Our approach is based on the celebrated Perron-Frobenius theorem and the comparison principle. Consequently, we get some new explicit criteria for global exponential stability of the zero solution of general nonlinear time-varying Volterra difference equations. Furthermore, we derive an explicit stability bound for equations subject to nonlinear time-varying perturbations. Finally, the obtained results are applied to study uniform attraction of equilibrium of discrete-time bidirectional associative memory neural networks. Some illustrative examples are given.

\section{Preliminaries}

In this section, we present notation and some preliminary results which will be of use in what follows. Let $\mathbb{R}$ be the set of all real numbers and let $\mathbb{N}$ be the set of all natural numbers. For any $k_{1}, k_{2} \in \mathbb{Z}, k_{1} \leq k_{2}$, let $\mathbb{Z}_{\left[k_{1}, k_{2}\right]}$ be the set of all integers in the interval $\left[k_{1}, k_{2}\right]$. Set $\mathbb{Z}_{+}:=\mathbb{N} \cup\{0\}$ and $\mathbb{Z}_{-}:=\left\{-k: k \in \mathbb{Z}_{+}\right\}$. Let $n, l, q$ be positive integers. Inequalities between real matrices and vectors will be understood component-wise, i.e. for two real $l \times q$-matrices $A=\left(a_{i j}\right)$ and $B=\left(b_{i j}\right)$, the inequality $A \geq B$ means $a_{i j} \geq b_{i j}$ for $i \in \mathbb{Z}_{[1, l]}, j \in \mathbb{Z}_{[1, q]}$. In particular, if $a_{i j}>b_{i j}$ for $i \in \mathbb{Z}_{[1, l]}$, $j \in \mathbb{Z}_{[1, q]}$, then we write $A \gg B$ instead of $A \geq B$. The set of all nonnegative $l \times q$-matrices is denoted by $\mathbb{R}_{+}^{l \times q}$. The identity $m \times m$-matrix will be denoted by $I_{m}$. If $x=\left(x_{1}, x_{2}, \ldots, x_{m}\right)^{T} \in \mathbb{R}^{m}$ and $P=\left(p_{i j}\right) \in \mathbb{R}^{l \times q}$ we define $|x|=\left(\left|x_{i}\right|\right)$ and $|P|=\left(\left|p_{i j}\right|\right)$. It is easy to see that $|C D|=|C||D|$. For any matrix $A \in \mathbb{R}^{m \times m}$, the spectral radius of $A$ is denoted by $\rho(A)=\max \{|z|: z \in \sigma(A)\}$, where $\sigma(A):=\left\{z \in \mathbb{C}: \operatorname{det}\left(z I_{m}-A\right)=0\right\}$ is the set of all eigenvalues of $A$. A norm $\|$.$\| on \mathbb{R}^{m}$ is said to be monotonic if $|x| \leq|y|$ implies $\|x\| \leq\|y\|$ for all $x, y \in \mathbb{R}^{m}$. Every $p$-norm 
on $\mathbb{R}^{m}\left(\|x\|_{p}=\left(\left|x_{1}\right|^{p}+\left|x_{2}\right|^{p}+\ldots+\left|x_{m}\right|^{p}\right)^{\frac{1}{p}}, 1 \leq p<\infty\right.$ and

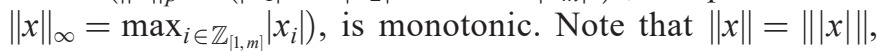
$\forall x \in \mathbb{R}^{m}$ for any monotonic norm.

Throughout this paper, the norm of vectors is assumed to be monotonic and the norm $\|M\|$ of a matrix $M \in \mathbb{R}^{l \times q}$ is always understood as the operator norm defined by $\|M\|=\max _{\|y\|=1}\|M y\|$, where $\mathbb{R}^{l}$ and $\mathbb{R}^{q}$ are provided with some monotonic vector norms. Recall that $\rho(M) \leq\|M\|$ for any matrix norm, see e.g. [7], and the operator norm $\|\cdot\|$ has the following monotonicity property, see e.g. [14],

$P \in \mathbb{R}^{l \times q}, Q \in \mathbb{R}_{+}^{l \times q},|P| \leq Q \Rightarrow\|P\| \leq\|P\|\|\| Q \|$.

The next theorem summarizes some basic properties of nonnegative matrices which will be used in what follows.

Theorem 2.1. [14]. Let $M \in \mathbb{R}_{+}^{m \times m}, t \in \mathbb{R}$, then

(i) (Perron-Frobenius) $\rho(M)$ is an eigenvalue of $M$ and there exists a nonnegative eigenvector $x \in \mathbb{R}^{m}, x \neq 0$ such that $M x=\rho(M) x$.

(ii) $\left(t I_{m}-M\right)^{-1}$ exists and is nonnegative if and only if $t>\rho(M)$.

The following theorem follows from Theorem 2.1.

Theorem 2.2. [20]. Let $M \in \mathbb{R}_{+}^{m \times m}$. Then the following statements are equivalent:

(i) $\rho(M)<1$;

(ii) $\exists p \in \mathbb{R}^{m}, p \gg 0: M p \ll p$;

(iii) $\left(I_{m}-M\right)^{-1} \geq 0$.

\section{Explicit criteria for exponential stability}

Consider a nonlinear Volterra difference equation of the form

$x(n+1)=F(n, x(n))+\sum_{k=0}^{n} G(n, k, x(k)), \quad n \geq n_{0}$,

where $F(\cdot, \cdot): \mathbb{Z}_{+} \times \mathbb{R}^{m} \rightarrow \mathbb{R}^{m}$ and $G(\cdot, \cdot, \cdot): \mathbb{Z}_{+} \times \mathbb{Z}_{+} \times \mathbb{R}^{m} \rightarrow \mathbb{R}^{m}$ are given functions such that $F(n, 0)=0$ for all $n \in \mathbb{Z}_{+}$and $G(n, k, 0)=0$ for $n \geq k, n, k \in \mathbb{Z}_{+}$(i.e. $\xi=0$ is an equilibrium point of (2)).

For given $n_{0} \in \mathbb{Z}_{+}$, denote $\mathscr{S}_{n_{0}}$ the set of all functions $\varphi(\cdot)$ : $\mathbb{Z}_{\left[0, n_{0}\right]} \rightarrow \mathbb{R}^{m}$. Let $\|\varphi\|_{n_{0}}=\max \left\{\|\varphi(n)\|: n \in \mathbb{Z}_{\left[0, n_{0}\right]}\right\}$, for each $\varphi(\cdot) \in \mathscr{S}_{n_{0}}$. Clearly, for fixed $n_{0} \in \mathbb{Z}_{+}$and given $\varphi \in \mathscr{S}_{n_{0}}$, (2) has a unique solution satisfying the initial condition

$$
x(n)=\varphi(n), \text { for all } n \in \mathbb{Z}_{\left[0, n_{0}\right]} .
$$

This solution is denoted by $x\left(\cdot, n_{0}, \varphi\right)$.

Definition 3.1. The zero solution of (2) is said to be globally exponentially stable (shortly, GES) if there exist $K>0$ and $\lambda \in(0,1)$ such that

$$
\left\|x\left(n, n_{0}, \varphi\right)\right\| \leq K \lambda^{n-n_{0}}\|\varphi\|_{n_{0}}
$$

for all $n, n_{0} \in \mathbb{Z}_{+}, n \geq n_{0}, \varphi(\cdot) \in \mathscr{S}_{n_{0}}$.
We are now in a position to prove the main result of this paper.

Theorem 3.2. Suppose there exist $A(\cdot): \mathbb{Z}_{+} \rightarrow \mathbb{R}_{+}^{m \times m}$ and $B(\cdot, \cdot): \mathbb{Z}_{+} \times \mathbb{Z}_{+} \rightarrow \mathbb{R}_{+}^{m \times m}$ such that

$$
|F(n, x)| \leq A(n)|x| \text { and }|G(n, k, x)| \leq B(n, k)|x|,
$$

for all $n, k \in \mathbb{Z}_{+}, n \geq k, x \in \mathbb{R}^{m}$. Then the zero solution of (2) is GES provided one of the following conditions holds:

(i) There exist $\alpha>1$ and $p \in \mathbb{R}_{+}^{m}, p \gg 0$ so that

$$
\left(A(n)+\sum_{k=0}^{n} B(n, k) \alpha^{n-k}\right) p \leq \alpha^{-1} p, \quad \forall n \in \mathbb{Z}_{+} .
$$

(ii) There exist $\beta>1$ and $A \in \mathbb{R}_{+}^{m \times m}, \rho(A)<1$ so that

$$
\left(A(n)+\sum_{k=0}^{n} B(n, k) \beta^{n-k}\right) \leq A, \quad \forall n \in \mathbb{Z}_{+} .
$$

(iii) There exists $\gamma>1$ so that

$$
\sup _{n \in \mathbb{Z}_{+}}\left(\|A(n)\|+\sum_{k=0}^{n}\|B(n, k)\| \gamma^{n-k}\right)<1 .
$$

Remark 3.3. Roughly speaking, (5) means that (2) is "bounded above" by the linear equation

$$
y(n+1)=A(n) y(n)+\sum_{k=0}^{n} B(n, k) y(k), \quad n \in \mathbb{Z}_{+} .
$$

On the other hand, as shown in the proof of Theorem 3.2, any one of conditions (6-8), ensures that (9) is GES. Therefore, Theorem 3.2 states that if (2) is "bounded above" by the linear Volterra equation (9) and (9) is GES (one of conditions (6-8), holds) then the zero solution of (2) is also GES.

Proof. We first prove that the zero solution of (2) is GES provided (i) holds. The proof consists of two steps.

Step 1. We show that

$$
\left\|x\left(n, n_{0}, \varphi\right)\right\| \leq K \lambda^{n-n_{0}}
$$

for all $n, n_{0} \in \mathbb{Z}_{+}, n \geq n_{0}, \varphi \in \mathscr{S}_{n_{0}},\|\varphi\|_{n_{0}} \leq 1$, for some $K>0$, $\lambda \in(0,1)$.

Since $p \gg 0$, there exists $M>1\left(M\right.$ is independent of $\left.n_{0}\right)$ such that

$$
\begin{aligned}
& M p \gg|\varphi(n)|, \quad \forall n \in \mathbb{Z}_{\left[0, n_{0}\right]}, \\
& \forall \varphi \in \mathscr{S}_{n_{0}}, \quad\|\varphi\|_{n_{0}} \leq 1 .
\end{aligned}
$$

Let us define $\lambda:=\alpha^{-1}$ and $u(n):=M \lambda^{n-n_{0}} p, n \in \mathbb{Z}$. From (3) and (10), it follows that

$$
u(n) \gg|x(n)|, \quad \forall n \in \mathbb{Z}_{\left[0, n_{0}\right]} .
$$


It is worth noticing that

$$
\begin{aligned}
\left|x\left(n_{0}+1\right)\right| & \stackrel{(2)}{=}\left|F\left(n_{0}, x\left(n_{0}\right)\right)+\sum_{k=0}^{n_{0}} G\left(n_{0}, k, x(k)\right)\right| \\
& \leq\left|F\left(n_{0}, x\left(n_{0}\right)\right)\right|+\sum_{k=0}^{n_{0}}\left|G\left(n_{0}, k, x(k)\right)\right| \\
& \stackrel{(5)}{\leq} A\left(n_{0}\right)\left|x\left(n_{0}\right)\right|+\sum_{k=0}^{n_{0}} B\left(n_{0}, k\right)|x(k)| \\
& \stackrel{(11)}{ } \leq A\left(n_{0}\right) M p+\sum_{k=0}^{n_{0}} B\left(n_{0}, k\right) M \lambda^{k-n_{0}} p \\
& =M\left(A\left(n_{0}\right)+\sum_{k=0}^{n_{0}} B\left(n_{0}, k\right) \alpha^{n_{0}-k}\right) p \\
& \stackrel{(6)}{\leq} M \alpha^{-1} p \\
& =u\left(n_{0}+1\right) .
\end{aligned}
$$

By induction, we can show that

$$
\begin{aligned}
& |x(n)|=\left|x\left(n, n_{0}, \varphi\right)\right| \leq M \lambda^{n-n_{0}} p, \\
& \forall n \geq n_{0}, \forall \varphi \in \mathscr{S}_{n_{0}},\|\varphi\|_{n_{0}} \leq 1 .
\end{aligned}
$$

By the monotonicity of vector norms, it follows that

$$
\left\|x\left(n, n_{0}, \varphi\right)\right\| \leq M\|p\| \lambda^{n-n_{0}}=K \lambda^{n-n_{0}},
$$

for all $n \geq n_{0}, \varphi \in \mathscr{S}_{n_{0}},\|\varphi\|_{n_{0}} \leq 1$, where $K:=M\|p\|$.

Step 2. We show that (4) holds

Consider the linear Volterra equation (9). By Step 1, we have for any $\varphi \in \mathscr{S}_{n_{0}},\|\varphi\|_{n_{0}} \leq 1$,

$$
\left\|y\left(n, n_{0}, \varphi\right)\right\| \leq K \lambda^{n-n_{0}}, \quad \forall n \geq n_{0},
$$

where $K:=M\|p\|$. By the linearity of (9),

$$
\left\|y\left(n, n_{0}, \frac{\varphi}{\|\varphi\|_{n_{0}}}\right)\right\|=\frac{1}{\|\varphi\|_{n_{0}}}\left\|y\left(n, n_{0}, \varphi\right)\right\| \leq K \lambda^{n-n_{0}}
$$

for all $n \geq n_{0}, \varphi \in \mathscr{S}_{n_{0}},\|\varphi\|_{n_{0}} \neq 0$.

Therefore,

$$
\begin{aligned}
& \left\|y\left(n, n_{0}, \varphi\right)\right\| \leq K \lambda^{n-n_{0}}\|\varphi\|_{n_{0}}, \\
& \forall n \geq n_{0}, \forall \varphi \in \mathscr{S}_{n_{0}} .
\end{aligned}
$$

For given $\varphi \in \mathscr{S}_{n_{0}}$, let $x(\cdot):=x\left(\cdot, n_{0}, \varphi\right)$ be the solution of $(2-3)$ and let $y(\cdot):=y\left(\cdot, n_{0},|\varphi|\right)$, where $|\varphi|(n):=|\varphi(n)|, n \in \mathbb{Z}_{\left[0, n_{0}\right]}$. Since $A(n) \geq 0, B(n, k) \geq 0$ for $n, k \in \mathbb{Z}_{+}, n \geq k$, and $|\varphi| \geq 0$, it follows that $y(n) \geq 0, \forall n \in \mathbb{Z}_{+}, n \geq n_{0}$.
Note that

$$
\begin{aligned}
\left|x\left(n_{0}+1\right)\right| & \leq\left|F\left(n_{0}, x\left(n_{0}\right)\right)\right|+\sum_{k=0}^{n_{0}}\left|G\left(n_{0}, k, x(k)\right)\right| \\
& \stackrel{(5)}{\leq} A\left(n_{0}\right)\left|x\left(n_{0}\right)\right|+\sum_{k=0}^{n_{0}} B\left(n_{0}, k\right)|x(k)| \\
& \stackrel{(3)}{=} A\left(n_{0}\right)\left|\varphi\left(n_{0}\right)\right|+\sum_{k=0}^{n_{0}} B\left(n_{0}, k\right)|\varphi(k)| \\
& \stackrel{(9)}{=} A\left(n_{0}\right) y\left(n_{0}\right)+\sum_{k=0}^{n_{0}} B\left(n_{0}, k\right) y(k) \\
& =y\left(n_{0}+1\right) .
\end{aligned}
$$

By induction, we have

$$
|x(n)| \leq y(n), \quad \forall n \in \mathbb{Z}_{+}
$$

By the monotonicity of vector norms,

$$
\|x(n)\|=\||x(n)|\| \leq\|y(n)\|, \quad \forall n \in \mathbb{Z}_{+} .
$$

Thus, (4) follows from (12) and (13). Hence, the zero solution of (2) is GES.

Next, we show that (ii) implies (i). Since $A \in \mathbb{R}_{+}^{m \times m}$, $\rho(A)<1$, there is a vector $p \in \mathbb{R}^{m}, p \gg 0$ such that $A p \ll p$, by Theorem 2.2. By continuity,

$$
A p \ll \eta^{-1} p
$$

for some $\eta>1$. Let $\beta$ be as in (ii) and let $\alpha_{0}:=\min \{\beta, \eta\}$. Clearly, $\alpha_{0}>1$ and

$$
\begin{aligned}
& \left(A(n)+\sum_{k=0}^{n} B(n, k) \alpha_{0}^{n-k}\right) \leq \\
& \left(A(n)+\sum_{k=0}^{n} B(n, k) \beta^{n-k}\right) \stackrel{(7)}{\leq} A .
\end{aligned}
$$

Therefore,

$$
\left(A(n)+\sum_{k=0}^{n} B(n, k) \alpha_{0}^{n-k}\right) p \leq A p \stackrel{(14)}{\leq} \eta^{-1} p \leq \alpha_{0}^{-1} p .
$$

Thus, (i) holds.

Finally, it remains to show that the zero solution of (2) is GES provided (iii) holds. From (8), it follows that $\|A(n)\|+$ $\sum_{k=0}^{n}\|B(n, k)\| \gamma^{n-k}<a, \forall n \in \mathbb{Z}_{+}$, for some $a \in[0,1)$. Thus, the zero solution of the scalar difference equation

$z(n+1)=\|A(n)\| z(n)+\sum_{k=0}^{n}\|B(n, k)\| z(k), n \in \mathbb{Z}_{+}$

is GES, by (ii). Fix $\varphi \in \mathscr{S}_{n_{0}}$. Let $x(\cdot):=x\left(\cdot, n_{0}, \varphi\right)$ be the solution of (2-3) and let $z(\cdot):=z\left(\cdot, n_{0},\|\varphi(\cdot)\|\right)$ be the solution of (15) 
with the initial value function $\|\varphi(\cdot)\|$. By a similar argument as in Step 2 of the proof of (i), we get

$$
\|x(n)\| \leq z(n), \quad \forall n \in \mathbb{Z}_{+} .
$$

Therefore, the zero solution of (2) is GES. This completes the proof.

For given $\gamma>1$, let us denote

$$
\begin{gathered}
l^{\gamma}\left(\mathbb{K}^{m \times m}\right):= \\
\left\{(B(n))_{n}: B(n) \in \mathbb{K}^{m \times m}, n \in \mathbb{Z}_{+}, \sum_{n=0}^{\infty}\|B(n)\| \gamma^{n}<+\infty\right\}, \\
\mathbb{K}:=\mathbb{R}, \mathbb{C} .
\end{gathered}
$$

In particular, the following follows from Theorem 3.2.

Theorem 3.4. Suppose there exist $A \in \mathbb{R}_{+}^{m \times m}$ and $B(\cdot): \mathbb{Z}_{+}$ $\rightarrow \mathbb{R}_{+}^{m \times m}$ such that

$$
|F(n, x)| \leq A|x| \text { and }|G(n, k, x)| \leq B(n-k)|x|
$$

for all $n, k \in \mathbb{Z}_{+}, n \geq k, x \in \mathbb{R}^{m}$. If $(B(n))_{n} \in l^{\gamma}\left(\mathbb{R}^{m \times m}\right)$ for some $\gamma>1$ and $\rho\left(A+\sum_{n=0}^{+\infty} B(n)\right)$, then the zero solution of (2) is GES.

Proof. Since $\rho\left(A+\sum_{k=0}^{+\infty} B(k)\right)<1$ and $(B(n))_{n} \in l^{\gamma}\left(\mathbb{R}^{m \times m}\right)$ with $\gamma>1, \rho\left(A+\sum_{k=0}^{+\infty} B(k) \beta^{k}\right)<1$, for some $\beta \in(1, \gamma]$, by continuity of the spectral radius, see [3]. Note that $A+\sum_{k=0}^{n} B(n-k)$ $\beta^{n-k}=A+\sum_{k=0}^{n} B(k) \beta^{k} \leq A+\sum_{k=0}^{+\infty} B(k) \beta^{k}, \forall n \in \mathbb{Z}_{+}$. Therefore, (ii) of Theorem 3.4 holds and the zero solution of (2) is GES.

We illustrate the obtained results by a couple of examples.

Example 3.5. Consider a scalar nonlinear Volterra difference equation

$$
\begin{aligned}
x(n+1) & =\frac{n e^{-x^{2}(n)} x(n)}{4 n+1} \\
& +\sum_{k=0}^{n} \frac{2 k^{2} \arctan \left(a^{n-k} x(k)\right)}{\left(k^{2}+1\right)(3(n-k)+1)(3(n-k)+4)},
\end{aligned}
$$

where $0<a<1$ and $n \in \mathbb{Z}_{+}$.

Let

$$
\begin{aligned}
& F(n, x)=\frac{n e^{-x^{2}} x}{4 n+1} \text { and } \\
& G(n, k, x)=\frac{2 k^{2} \arctan \left(a^{n-k} x\right)}{\left(k^{2}+1\right)(3(n-k)+1)(3(n-k)+4)}
\end{aligned}
$$

where $n, k \in \mathbb{Z}_{+}, n \geq k$ and $x \in \mathbb{R}$. Since

$$
\left|\frac{n e^{-x^{2}} x}{4 n+1}\right| \leq \frac{1}{4}|x| \text { and } \quad\left|\frac{k^{2} \arctan \left(a^{n-k} x\right)}{k^{2}+1}\right| \leq a^{n-k}|x|
$$

for all $x \in \mathbb{R}, n, k \in \mathbb{Z}_{+}, n \geq k$, it follows that

$$
|F(n, x)| \leq A|x| \text { and }|G(n, k, x)| \leq B(n-k)|x|
$$

for all $x \in \mathbb{R}, n, k \in \mathbb{Z}_{+}, n \geq k$, where $A=\frac{1}{4}$ and $B(n)=\frac{2 a^{n}}{(3 n+1)(3 n+4)}, n \in \mathbb{Z}_{+}$.

Clearly,

$\left|A+\sum_{n=0}^{+\infty} B(n)\right| \leq \frac{1}{4}+\sum_{n=0}^{+\infty} \frac{2}{(3 n+1)(3 n+4)}=\frac{1}{4}+\frac{2}{3}<1$

and $|B(n)| \leq 1 / 2 a^{n}$ for all $n \in \mathbb{Z}_{+}$. Choosing $\gamma \in\left(1, a^{-1}\right)$, we have $a \gamma \in(0,1)$ and $\sum_{n=0}^{+\infty}|B(n)| \gamma^{n}<+\infty$. Thus, the zero solution of (18) is GES, by Theorem 3.4.

Example 3.6. Consider a nonlinear Volterra difference equation in $\mathbb{R}^{3}$ given by

$$
x(n+1)=F(n, x(n))+\sum_{k=0}^{n} G(n, k, x(k)),
$$

where

$$
F(n, x)=\left(\begin{array}{c}
\ln \left(1+\frac{0.1 n}{n+1}\left|x_{1}\right|+0.3 e^{-n}\left|x_{2}\right|\right) \\
0.25 x_{2} \sin \left(n x_{3}\right) \\
\sqrt{\left(\frac{0.6 n}{n^{2}+1} x_{1}\right)^{2}+\left(0.2 x_{3}\right)^{2}}
\end{array}\right)
$$

and

$$
G(n, k, x)=\left(\begin{array}{c}
\arctan \left(\frac{2^{k-n}}{(k+5)(k+6)} x_{1}+4^{k-n-2} x_{2}\right) \\
\ln \left(1+0.2 \times 3^{k-n}\left|x_{1}\right|\right) \\
4^{k-n-2} x_{1}+\frac{2^{k-n}}{(5 k+1)(5 k+6)} x_{3}
\end{array}\right)
$$

with $n, k \in \mathbb{Z}_{+}, n \geq k$ and $x:=x\left(x_{1}, x_{2}, x_{3}\right)^{T} \in \mathbb{R}^{3}$.

Define

$$
A(n):=\left(\begin{array}{ccc}
\frac{0.1 n}{n+1} & 0.3 e^{-n} & 0 \\
0 & 0.25 & 0 \\
\frac{0.6 n}{n^{2}+1} & 0 & 0.2
\end{array}\right)
$$

$$
B(n, k):=\left(\begin{array}{ccc}
\frac{2^{k-n}}{(k+5)(k+6)} & 4^{k-n-2} & 0 \\
0.2 \times 3^{k-n} & 0 & 0 \\
4^{k-n-2} & 0 & \frac{2^{k-n}}{(5 k+1)(5 k+6)}
\end{array}\right)
$$


with $n, k \in \mathbb{Z}_{+}, n \geq k$. It is easy to check that

$$
|F(n, x)| \leq A(n)|x| \text { and }|G(n, k, x)| \leq B(n, k)|x| \text {, }
$$

for all $n, k \in \mathbb{Z}_{+}, n \geq k, x=\mathbb{R}^{3}$. Thus, (5) holds. Furthermore, we have for $\beta=2$ that

$$
\begin{aligned}
& A(n)+\sum_{k=0}^{n} B(n, k) \beta^{n-k}= \\
& \left(\begin{array}{ccc}
0.1 \frac{n}{n+1}+ & 0.3 e^{-n}+0.25 \times & 0 \\
\sum_{k=0}^{n} \frac{1}{(k+5)(k+6)} & \sum_{k=0}^{n}\left(\frac{1}{2}\right)^{n-k} & \\
0.2 \sum_{k=0}^{n}\left(\frac{2}{3}\right)^{n-k} & 0.25 & 0 \\
0.6 \frac{n}{n^{2}+1}+0.25 \times & 0 & 0.2+ \\
\sum_{k=0}^{n}\left(\frac{1}{2}\right)^{n-k} & & \sum_{k=0}^{n} \frac{1}{(5 k+1)(5 k+6)}
\end{array}\right) \\
& \leq\left(\begin{array}{ccc}
0.1+ & 0.3+0.25 \times & 0 \\
\left(\frac{1}{5}-\frac{1}{n+6}\right) & 2\left(1-\left(\frac{1}{2}\right)^{n+1}\right) & \\
0.2 \times 3\left(1-\left(\frac{2}{3}\right)^{n+1}\right) & 0.25 & 0 \\
0.3+0.25 \times & 0 & 0.2+0.2 \times \\
2\left(1-\left(\frac{1}{2}\right)^{n+1}\right) & & \left(1-\frac{1}{(5 n+6)}\right)
\end{array}\right),
\end{aligned}
$$

for all $n, k \in \mathbb{Z}_{+}, n \geq k$.

Thus,

$$
A(n)+\sum_{k=0}^{n} B(n, k) \beta^{n-k} \leq A:=\left(\begin{array}{ccc}
0.3 & 0.8 & 0 \\
0.6 & 0.25 & 0 \\
0.8 & 0 & 0.4
\end{array}\right)
$$

for all $n, k \in \mathbb{Z}_{+}, n \geq k$ and $\rho(A)<1$. Therefore, the zero solution of (21) is GES, by (ii) of Theorem 3.2.

\section{Stability of perturbed equations}

Suppose (17) holds and $(B(n))_{n} \in l^{\gamma}\left(\mathbb{R}^{m \times m}\right)$ for some $\gamma>1$ and $\rho\left(A+\sum_{n=0}^{+\infty} B(n)\right)<1$. Thus, the zero solution of (2) is GES, by Theorem 3.4. Consider a perturbed equation of the form

$$
\begin{aligned}
x(n+1) & =(F(n, x(n))+\widetilde{F}(n, x(n)))+ \\
& +\sum_{k=0}^{n}(G(n, k, x(k))+\widetilde{G}(n, k, x(k))) .
\end{aligned}
$$

Here $F(\cdot, \cdot)$ and $G(\cdot, \cdot, \cdot)$ are as in $(2)$ whereas $\tilde{F}(\cdot, \cdot): \mathbb{Z}_{+} \times \mathbb{R}^{m} \rightarrow \mathbb{R}^{m}$ and $\widetilde{G}(\cdot, \cdot, \cdot): \mathbb{Z}_{+}^{2} \times \mathbb{R}^{m} \rightarrow \mathbb{R}^{m}$ are perturbations. Furthermore, we assume that

$$
\left\{\begin{array}{l}
|\widetilde{F}(n, x)| \leq D_{0} \Delta_{F} E_{0}|x| \\
|\widetilde{G}(n, k, x)| \leq D(n-k) \Delta_{G}(n-k) E(n-k)|x|
\end{array}\right.
$$

for all $n, k \in \mathbb{Z}_{+}, n \geq k, x \in \mathbb{R}^{m}$, where $D_{0} \in \mathbb{R}_{+}^{m \times l}, E_{0} \in \mathbb{R}_{+}^{q \times m}$, $D(\cdot): \mathbb{Z}_{+} \rightarrow \mathbb{R}_{+}^{m \times r}, E(\cdot): \mathbb{Z}_{+} \rightarrow \mathbb{R}_{+}^{s \times m}$ are known and $\Delta_{F} \in \mathbb{R}_{+}^{l \times q}$, $\Delta_{G}(\cdot): \mathbb{Z}_{+} \rightarrow \mathbb{R}_{+}^{r \times s}$ are unknown.

The main problem here is to seek a positive number, say $\eta$, such that the zero solution of an arbitrary perturbed equation of the form (22) remains GES whenever the size of $\left(\Delta_{F}, \Delta_{G}\right)$ is strictly less than $\eta$.

Theorem 4.1. Assume that $\left(\Delta_{G}(n)\right)_{n} \in l^{\gamma_{0}}\left(\mathbb{R}^{m \times m}\right)$ for some $\gamma_{0}>1$ and

$$
\max \left\{\sup _{n \in \mathbb{Z}_{+}}\|D(n)\|, \sup _{n \in \mathbb{Z}_{+}}\|E(n)\|\right\}<+\infty .
$$

If

$$
\begin{aligned}
& \left\|\left(\Delta_{F}, \Delta_{G}\right)\right\|< \\
& <\frac{1}{\sup _{P \in \Xi, Q \in \Omega}\left\{\left\|P\left(I_{m}-A-\sum_{k=0}^{+\infty} B(k)\right)^{-1} Q\right\|\right\}},
\end{aligned}
$$

where $\left\|\left(\Delta_{F}, \Delta_{G}\right)\right\|:=\left\|\Delta_{F}\right\|+\sum_{k=0}^{+\infty}\left\|\Delta_{G}(k)\right\|, \Xi:=\left\{E_{0}, E(0), E(1)\right.$, $E(2), \ldots\}$ and $\Omega:=\left\{D_{0}, D(0), D(1), D(2), \ldots\right\}$, then the zero solution of (22) remains GES.

Proof. By (17) and (23), it follows that

$$
|F(n, x)+\widetilde{F}(n, x)| \leq\left(A+D_{0} \Delta_{F} E_{0}\right)|x|
$$

and

$$
\begin{aligned}
& |(G(n, k, x)+\widetilde{G}(n, k, x))| \\
& \leq\left(B(n-k)+D(n-k) \Delta_{G}(n-k) E(n-k)\right)|x|,
\end{aligned}
$$

for all $n, k \in \mathbb{Z}_{+}, n \geq k, x \in \mathbb{R}^{m}$. Invoking (24) and $(B(n))_{n} \in$ $l^{\gamma}\left(\mathbb{R}^{m \times m}\right),\left(\Delta_{G}(n)\right)_{n} \in l^{\gamma_{0}}\left(\mathbb{R}^{m \times m}\right)$, we have $\left(B(n)+D(n) \Delta_{G}(n) E(n)\right)_{n}$ $\in l^{\gamma_{1}}\left(\mathbb{R}^{m \times m}\right)$, with $\gamma_{1}:=\min \left\{\gamma, \gamma_{0}\right\}$.

We show that $\rho\left(A+D_{0} \Delta_{F} E_{0}+\sum_{k=0}^{+\infty}\left(B(k)+D(k) \Delta_{G}(k)\right.\right.$ $E(k)))<1$ and then the zero solution of the perturbed equation (22) is GES by Theorem 3.4 .

Let $M:=A+\sum_{k=0}^{+\infty} B(k)$. Since $M, D_{0}, \Delta_{F}, E_{0}, D(k), \Delta_{G}(k)$, $E(k), k \in \mathbb{Z}_{+}$, are nonnegative, so is $M+D_{0} \Delta_{F} E_{0}+\sum_{k=0}^{+\infty} D(k)$ $\Delta_{G}(k) E(k)$. Assume on the contrary that $\rho_{0}:=\rho\left(M+D_{0} \Delta_{F} E_{0}+\right.$ $\left.\sum_{k=0}^{+\infty} D(k) \Delta_{G}(k) E(k)\right) \geq 1$. By the Perron-Frobenius Theorem (Theorem $2.1(i)$ ), there exists $x \in \mathbb{R}_{+}^{m}, x \neq 0$, such that

$$
\left(M+D_{0} \Delta_{F} E_{0}+\sum_{k=0}^{+\infty} D(k) \Delta_{G}(k) E(k)\right) x=\rho_{0} x,
$$

or equivalently,

$$
\left(\rho_{0} I_{m}-M\right) x=\left(D_{0} \Delta_{F} E_{0}+\sum_{k=0}^{+\infty} D(k) \Delta_{G}(k) E(k)\right) x .
$$


Let $H(t):=\left(t I_{m}-M\right), t \in \mathbb{R}$. Since $\rho(M)<1 \leq \rho_{0}, H\left(\rho_{0}\right)^{-1}$ and $H(1)^{-1}$ exist and are nonnegative, by Theorem 1.1 (ii). From (26), it follows that

$$
H\left(\rho_{0}\right)^{-1}\left(D_{0} \Delta_{F} E_{0}+\sum_{k=0}^{+\infty} D(k) \Delta_{G}(k) E(k)\right) x=x .
$$

Since $x \neq 0$ and $\sup _{n \in \mathbb{Z}_{+}}\|E(n)\|<+\infty,(27)$ implies

$$
0<\max \left\{\left\|E_{0} x\right\|, \sup _{n \in \mathbb{Z}_{+}}\|E(n) x\|\right\}<+\infty
$$

If $\max \left\{\left\|E_{0} x\right\|, \sup _{n \in \mathbb{Z}_{+}}\|E(n) x\|\right\}=\left\|E_{0} x\right\|$, multiplying both sides of (27) from the left by $E_{0}$, we get

$$
\begin{aligned}
& \left(E_{0} H\left(\rho_{0}\right)^{-1} D_{0} \Delta_{F} E_{0}\right) x+ \\
& +\left(\sum_{k=0}^{+\infty} E_{0} H\left(\rho_{0}\right)^{-1} D(k) \Delta_{G}(k) E(k)\right) x=E_{0} x .
\end{aligned}
$$

Taking norms both sides of the last equation, we have

$$
\begin{aligned}
& \left\|E_{0} H\left(\rho_{0}\right)^{-1} D_{0}\right\| \cdot\left\|\Delta_{F}\right\| \cdot\left\|E_{0} x\right\|+ \\
& +\sum_{k=0}^{+\infty}\left\|E_{0} H\left(\rho_{0}\right)^{-1} D(k)\right\| \cdot\left\|\Delta_{G}(k)\right\| \cdot\|E(k) x\| \geq\left\|E_{0} x\right\| .
\end{aligned}
$$

This implies

$$
\sup _{P \in \Xi, Q \in \Omega}\left\|P H\left(\rho_{0}\right)^{-1} Q\right\|\left(\left\|\Delta_{F}\right\|+\sum_{k=0}^{+\infty}\left\|\Delta_{G}(k)\right\|\right)\left\|E_{0} x\right\| \geq\left\|E_{0} x\right\|,
$$

or equivalently,

$$
\sup _{P \in \Xi, Q \in \Omega}\left\|P H\left(\rho_{0}\right)^{-1} Q\right\| \cdot\left\|\left(\Delta_{F}, \Delta_{G}\right)\right\| \geq 1 .
$$

On the other hand, the resolvent identity gives

$$
H(1)^{-1}-H\left(\rho_{0}\right)^{-1}=\left(\rho_{0}-1\right) H\left(\rho_{0}\right)^{-1} H(1)^{-1} \geq 0 .
$$

This yields $H(1)^{-1} \geq H\left(\rho_{0}\right)^{-1} \geq 0$. Therefore, $P H(1)^{-1} Q \geq$ $P H\left(\rho_{0}\right)^{-1} Q \geq 0$, for any $P \in \Xi, Q \in \Omega$. By (1), $\left\|P H(1)^{-1} Q\right\| \geq$ $\left\|P H\left(\rho_{0}\right)^{-1} Q\right\| \geq 0$, for any $P \in \Xi, Q \in \Omega$. It follows from (28) that

$$
\begin{aligned}
& \left\|\left(\Delta_{F}, \Delta_{G}\right)\right\| \geq \frac{1}{\sup _{P \in \Xi, Q \in \Omega}\left\|P H(1)^{-1} Q\right\|} \\
& =\frac{1}{\sup _{P \in \Xi, Q \in \Omega}\left\{\left\|P\left(I_{m}-A-\sum_{k=0}^{+\infty} B(k)\right)^{-1} Q\right\|\right\}} .
\end{aligned}
$$

However, this conflicts with (25).
If $\sup _{n \in \mathbb{Z}_{+}}\|E(n) x\|>\left\|E_{0} x\right\|$, then for given $\varepsilon>0$, there is $n_{0} \in \mathbb{Z}_{+}$such that $\left\|E\left(n_{0}\right) x\right\|>\sup _{n \in \mathbb{Z}_{+}}\|E(n) x\|-\varepsilon>0$. Multiplying both sides of (27) from the left by $E\left(n_{0}\right)$, we get

$$
\begin{aligned}
& \left(E\left(n_{0}\right) H\left(\rho_{0}\right)^{-1} D_{0} \Delta_{F} E_{0}\right) x \\
& +\left(\sum_{k=0}^{+\infty} E\left(n_{0}\right) H\left(\rho_{0}\right)^{-1} D(k) \Delta_{G}(k) E(k)\right) x=E\left(n_{0}\right) x .
\end{aligned}
$$

Taking norms, we get

$$
\begin{aligned}
& \left\|E\left(n_{0}\right) H\left(\rho_{0}\right)^{-1} D_{0}\right\| \cdot\left\|\Delta_{F}\right\| \cdot\left\|E_{0} x\right\|+ \\
& \sum_{k=0}^{+\infty}\left\|E\left(n_{0}\right) H\left(\rho_{0}\right)^{-1} D(k)\right\| \cdot\left\|\Delta_{G}(k)\right\|\|E(k) x\| \geq\left\|E\left(n_{0}\right) x\right\| .
\end{aligned}
$$

It follows that

$$
\begin{aligned}
& \sup _{P \in \Xi, Q \in \Omega}\left\|P H\left(\rho_{0}\right)^{-1} Q\right\|\left(\left\|\Delta_{F}\right\|+\sum_{k=0}^{+\infty}\left\|\Delta_{G}(k)\right\|\right) \\
& \left(\left\|E\left(n_{0}\right) x\right\|+\varepsilon\right) \geq\left\|E\left(n_{0}\right) x\right\| .
\end{aligned}
$$

Since $\varepsilon>0$ is arbitrary, we derive

$$
\sup _{P \in \Xi, Q \in \Omega}\left\|P H\left(\rho_{0}\right)^{-1} Q\right\|\left(\left\|\Delta_{F}\right\|+\sum_{k=0}^{+\infty}\left\|\Delta_{G}(k)\right\|\right) \geq 1 .
$$

By a similar argument as in the preceding paragraph, we get (29). This is a contradiction which completes the proof.

Example 4.2. We now reconsider the Volterra difference equation defined by (18-19). As shown in Example 3.5, the zero solution of (18-19) is GES. Consider a perturbed equation given by

$$
\begin{aligned}
x(n+1) & =(F(n, x(n))+\widetilde{F}(n, x(n)))+ \\
& +\sum_{k=0}^{n}(G(n, k, x(k))+\widetilde{G}(n, k, x(k))),
\end{aligned}
$$

where $\widetilde{F}(\cdot, \cdot)$ and $\widetilde{G}(\cdot, \cdot, \cdot)$ are given by

$$
\widetilde{F}(n, x)=\frac{e^{-\sin ^{2}(n x)} u(n) x}{5}
$$

and

$$
\widetilde{G}(n, k, x)=\frac{(n-k)^{2} \cos k \ln (1+|v(n-k) x|)}{3(n-k)^{2}+1},
$$

where $x \in \mathbb{R}, n, k \in \mathbb{Z}_{+}, n \geq k ; u(\cdot): \mathbb{Z}_{+} \rightarrow \mathbb{R}$ and $v(\cdot): \mathbb{Z}_{+} \rightarrow \mathbb{R}$ are unknown functions satisfying $\sup _{n \in \mathbb{Z}_{+}}|u(n)|<+\infty$ and $(v(n))_{n} \in l^{\gamma}(\mathbb{R})$ for some $\gamma>1$. It is clear that 


$$
\begin{aligned}
& |\widetilde{F}(n, x)| \leq \frac{1}{5}|u(n)||x|,|\widetilde{G}(n, k, x)| \leq \\
& \leq \frac{(n-k)^{2}}{3(n-k)^{2}+1}|v(n-k)||x|,
\end{aligned}
$$

for all $x \in \mathbb{R}, n, k \in \mathbb{Z}_{+}, n \geq k$.

By Theorem 4.1, the zero solution of (30) is GES provided

$$
\begin{aligned}
& \sup _{n \in \mathbb{Z}_{+}}|u(n)|+\sum_{n=0}^{+\infty}|v(n)|< \\
& <\frac{1}{\sup _{n \in \mathbb{Z}_{+}}\left\{\left(1-\frac{1}{4}-\sum_{k=0}^{+\infty} \frac{2 a^{k}}{(3 k+1)(3 k+4)}\right)^{-1} \frac{n^{2}}{3 n^{2}+1}\right\}},
\end{aligned}
$$

or equivalently,

$$
\sup _{n \in \mathbb{Z}_{+}}|u(n)|+\sum_{n=0}^{+\infty}|v(n)|<\frac{1}{\left(\frac{3}{4}-p\right)^{-1} \frac{1}{3}}=\frac{9}{4}-3 p,
$$

where $p:=\sum_{k=0}^{+\infty} \frac{2 a^{k}}{(3 k+1)(3 k+4)} \in(0,2 / 3]$ and $a \in(0,1)$.

\section{An application}

In this section, we apply the obtained results to study uniform attraction of equilibrium of discrete-time bidirectional associative memory (BAM) neural networks, see e.g. [18, 24].

Consider a discrete-time BAM neural network with infinite delay in $\mathbb{R}^{2}$ described by

$$
\left\{\begin{array}{l}
x_{1}(n+1)=a_{1}(n) x_{1}(n) \\
+b_{1}(n) f_{1}\left(\sum_{k=0}^{+\infty} g_{1}(k) x_{2}(n-k)\right)+I_{1}(n), \\
x_{2}(n+1)=a_{2}(n) x_{2}(n) \\
+b_{2}(n) f_{2}\left(\sum_{k=0}^{+\infty} g_{2}(k) x_{1}(n-k)\right)+I_{2}(n), n \in \mathbb{Z}_{+},
\end{array}\right.
$$

with the initial condition

$$
x_{1}(k)=\psi_{1}(k) ; x_{2}(k)=\psi_{2}(k), \forall k \in \mathbb{Z}_{-},
$$

where $\psi_{1}(\cdot), \psi_{2}(\cdot): \mathbb{Z}_{-} \rightarrow \mathbb{R}$ are given bounded functions.

Throughout, we assume that

$\left(\mathrm{H}_{1}\right)$ There exist $a_{i}, b_{i} \in \mathbb{R}_{+}, i \in\{1,2\}$ such that $\left|a_{i}(n)\right| \leq a_{i}$ and $\left|b_{i}(n)\right| \leq b_{i}, \forall n \in \mathbb{Z}_{+}, i \in\{1,2\} ;$

$\left(\mathrm{H}_{2}\right)$ There exists $c_{i} \in \mathbb{R}_{+}, i \in\{1,2\}$ such that $\left|f_{i}(x)-f_{i}(y)\right| \leq c_{i}|x-y|, i \in\{1,2\}$, for all $x, y \in \mathbb{R} ;$ $\left(\mathrm{H}_{3}\right)\left(g_{i}(n)\right)_{n} \in l^{\gamma}(\mathbb{R})$, for some $\gamma>1, i \in\{1,2\}$.
Suppose $x^{*}:=\left(x_{1}^{*}, x_{2}^{*}\right)^{T} \in \mathbb{R}^{2}$ is an equilibrium of the BAM neural network (33). That is,

$$
\left\{\begin{array}{l}
x_{1}^{*}=a_{1}(n) x_{1}^{*}+b_{1}(n) f_{1}\left(\sum_{k=0}^{+\infty} g_{1}(k) x_{2}^{*}\right)+I_{1}(n), n \in \mathbb{Z}_{+}, \\
x_{2}^{*}=a_{2}(n) x_{2}^{*}+b_{2}(n) f_{2}\left(\sum_{k=0}^{+\infty} g_{2}(k) x_{1}^{*}\right)+I_{2}(n), n \in \mathbb{Z}_{+} .
\end{array}\right.
$$

The following theorem gives an explicit criterion for uniform attraction of the equilibrium of (33).

Theorem 5.1. Let $\left(\mathrm{H}_{1}-\mathrm{H}_{3}\right)$ hold. If

$$
\begin{aligned}
\min \left\{\max _{i \in\{1,2\}}\left\{a_{i}+b_{i} c_{i} \sum_{k=0}^{+\infty} g_{i}(k)\right\},\right. \\
\left.\max _{i, j \in\{1,2\}, i \neq j}\left\{a_{i}+b_{j} c_{j} \sum_{k=0}^{+\infty} g_{j}(k)\right\}\right\}<1 .
\end{aligned}
$$

then the equilibrium point $x^{*}$ of (33) is uniformly attractive, i.e, $\left\|x(n)-x^{*}\right\| \rightarrow 0$ as $n \rightarrow+\infty$, where $x(n)=\left(x_{1}(n), x_{2}(n)\right)^{T} \in \mathbb{R}^{2}$, $n \in \mathbb{Z}_{+}$.

Proof. Let $y_{i}(n):=x_{i}(n)-x_{i}^{*}, i \in\{1,2\}, n \in \mathbb{Z}$. It follows from (33-34) that

$$
\left\{\begin{array}{l}
y_{1}(n+1)=a_{1}(n) y_{1}(n)+b_{1}(n) \times \\
\left(f_{1}\left(\sum_{k=0}^{+\infty} g_{1}(k)\left[y_{2}(n-k)+x_{2}^{*}\right]\right)-f_{1}\left(\sum_{k=0}^{+\infty} g_{1}(k) x_{2}^{*}\right)\right), \\
y_{2}(n+1)=a_{2}(n) y_{2}(n)+b_{2}(n) \times \\
\left(f_{2}\left(\sum_{k=0}^{+\infty} g_{2}(k)\left[y_{1}(n-k)+x_{1}^{*}\right]\right)-f_{2}\left(\sum_{k=0}^{+\infty} g_{2}(k) x_{1}^{*}\right)\right) .
\end{array}\right.
$$

Clearly, (36) takes the form

$$
y(n+1)=A(n) y(n)+B(n) F\left(\sum_{k=-\infty}^{n} G(n-k) y(k)\right),
$$

where $y(n):=\left(y_{1}(n), y_{2}(n)\right)^{T} \in \mathbb{R}^{2}, n \in \mathbb{Z}_{+}$, and for each $n \in \mathbb{Z}_{+}$,

$$
\begin{aligned}
A(n) & :=\left(\begin{array}{cc}
a_{1}(n) & 0 \\
0 & a_{2}(n)
\end{array}\right), B(n):=\left(\begin{array}{cc}
b_{1}(n) & 0 \\
0 & b_{2}(n)
\end{array}\right), \\
G(n) & :=\left(\begin{array}{cc}
0 & g_{1}(n) \\
g_{2}(n) & 0
\end{array}\right),
\end{aligned}
$$

and

$$
\begin{aligned}
F(z) & :=\left(\begin{array}{c}
f_{1}\left(z_{1}+\sum_{k=0}^{+\infty} g_{1}(k) x_{2}^{*}\right)-f_{1}\left(\sum_{k=0}^{+\infty} g_{1}(k) x_{2}^{*}\right) \\
f_{2}\left(z_{2}+\sum_{k=0}^{+\infty} g_{2}(k) x_{1}^{*}\right)-f_{2}\left(\sum_{k=0}^{+\infty} g_{2}(k) x_{1}^{*}\right)
\end{array}\right), \\
z & :=\left(z_{1}, z_{2}\right)^{T} \in \mathbb{R}^{2} .
\end{aligned}
$$


Let $A:=\operatorname{diag}\left(a_{1}, a_{2}\right)$ and $D:=\operatorname{diag}\left(b_{1} c_{1}, b_{2} c_{2}\right) \in \mathbb{R}^{2 \times 2}$ and

$M:=A+\sum_{k=0}^{+\infty} D G(k)=\left(\begin{array}{cc}a_{1} & b_{1} c_{1} \sum_{k=0}^{+\infty} g_{1}(k) \\ b_{2} c_{2} \sum_{k=0}^{+\infty} g_{2}(k) & a_{2}\end{array}\right)$.

Suppose $\mathbb{R}^{2}$ is endowed with $\|\cdot\|_{1}$ or $\|\cdot\|_{\infty}$, then $\|E\|_{1}=\max _{1 \leq j \leq 2}$ $\sum_{i=1}^{2}\left|e_{i j}\right|$ and $\|E\|_{\infty}=\max _{1 \leq i \leq 2} \sum_{j=1}^{2}\left|e_{i j}\right|$, for $E=\left(e_{i j}\right) \in \mathbb{R}^{2 \times 2}$, see e.g. [7]. Note that (35) implies that $\rho(M) \leq \min \left\{\|M\|_{\infty},\|M\|_{1}\right\}<1$. Since $\rho(M)<1$ and $(G(n))_{n} \in l^{\gamma}\left(\mathbb{R}^{2 \times 2}\right)$, it follows that the zero solution of the linear time-invariant equation

$$
z(n+1)=A z(n)+\sum_{k=0}^{n} D G(n-k) z(k), \quad n \in \mathbb{Z}_{+},
$$

is GES, by Theorem 3.4.

Next, consider the linear time-invariant equation with infinite delay

$$
z(n+1)=A z(n)+\sum_{k=-\infty}^{n} D G(n-k) z(k), \quad n \in \mathbb{Z}_{+},
$$

with the initial condition $z(s)=\psi(s), s \in \mathbb{Z}_{-}$, where $\psi(\cdot): \mathbb{Z}_{-}$ $\rightarrow \mathbb{R}^{2}$ is bounded. Then (39) can be rewritten as

$$
z(n+1)=A z(n)+\sum_{k=0}^{n} D G(n-k) z(k)+g(n), n \in \mathbb{Z}_{+},(
$$

where $g(n):=\sum_{j=-\infty}^{-1} D G(n-j) \psi(j), n \in \mathbb{Z}_{+}$. Since (38) is GES, the kernel $G(\cdot)$ and the resolvent of (38) exponentially decay, see e.g. [8]. Using the variation of constants formula $[10]$, it is easy to see that

$$
\|z(n)\| \rightarrow 0 \text { as } n \rightarrow+\infty,
$$

for any bounded initial function $\psi(\cdot): \mathbb{Z}_{-} \rightarrow \mathbb{R}^{2}$. Furthermore, invoking $\left(\mathrm{H}_{1}-\mathrm{H}_{3}\right)$, it is easy to show that

$$
|y(n)| \leq z(n), \quad \forall n \in \mathbb{Z}_{+},
$$

where $y(\cdot)$ is the unique solution of (37) with the initial function $\psi(\cdot):=\left(\psi_{1}(\cdot), \psi_{2}(\cdot)\right)^{T}$ and $z(\cdot)$ is the unique solution of (40) with the initial function $|\psi(\cdot)|:=\left(\left|\psi_{1}(\cdot)\right|,\left|\psi_{2}(\cdot)\right|\right)^{T}$. By the monotonicity of vector norm, we obtain

$$
\|y(n)\| \leq\|z(n)\|, \quad \forall n \in \mathbb{Z}_{+} .
$$

It follows from (41) and (42) that $\|y(n)\|=\left\|x(n)-x^{*}\right\| \rightarrow 0$ as $n \rightarrow+\infty$. This completes the proof.

Acknowledgements. This work was partially supported by Dong Thap university under the grant number CS.2015.01.26.

\section{REFERENCES}

[1] J.A.D. Appleby, I. Gyori, and D.W. Reynolds, "On exact convergence rates for solutions of linear systems of Volterra difference equations", J. Difference Equ. Appl. 12, 1257-1275 (2006).
[2] H. Brunner, and P.J. Houwen, The Numerical Solution of Volterra Equations, CWI. Monographs, North-Holland, Amsterdam, 1986.

[3] L. Burlando, "Continuity of spectrum and spectral radius in algebras of operators", Ann. Fac. Sci. Toulouse Math. 9, 5-54 (1988).

[4] M.R. Crisci, V.B. Kolmanovskii, E. Russo, and A. Vecchio, "Stability of difference Volterra equations: direct Liapunov method and numerical procedure", Comput. Math. Appl. 36, 77-97 (1998).

[5] M.R. Crisci, V.B. Kolmanovskii, E. Russo, and A. Vecchio, "On the exponential stability of discrete Volterra equations", J. Difference Equ. Appl. 6, 667-680 (2000).

[6] C. Cuevas, F. Dantas, M. Choquehuanca, and H. Soto, "Boundedness properties for Volterra difference equations", Appl. Math. Comput. 219 , 6986-6999 (2013).

[7] S. Elaydi, An Introduction to Difference Equations, Springer Verlag, 2005.

[8] S. Elaydi, and S. Murakami, "Asymptotic stability versus exponential stability in linear Volterra difference equations of convolution type", J. Difference Equ. Appl. 2, 401-410 (1996).

[9] S. Elaydi, and V. Kocic, "Global stability of a nonlinear Volterra difference equations”, Diff. Eqns. Dyn. Sys. 2, 337-345 (1994).

[10] S. Elaydi, "Stability and asymptoticity of Volterra difference equations: A progress report", J. Comput. Appl. Math. 228, 504-513 (2009).

[11] P.W. Eloe, M.N. Islam, and Y.N. Raffoul, "Uniform asymptotic stability in nonlinear Volterra discrete equations", Comput. Math. Appl. 45, 1033-1039 (2003).

[12] G. Gripenberg., S.O. Londen, and O. Staffans, Volterra integral and functional equations, Cambridge University Press, Vol. 34, 1990.

[13] I. Gyori, and D.W. Reynolds, "On admissibility of the resolvent of discrete Volterra equations", J. Difference Equ. Appl. 16, 1393-1412 (2010).

[14] D. Hinrichsen, and N.K. Son, "Stability radii of positive discrete-time equations under affine parameter perturbations", Internat. J. Robust Nonlinear Control 8, 1169-1188 (1988).

[15] V.B. Kolmanovskii, E. Castellanos-Velasco, and J. A. TorresMunoz, "A survey: stability and boundedness of Volterra difference equations", Nonlinear Anal. 53, 861-928 (2003).

[16] J.J. Levin, and J.A. Nohel, "The integrodifferential equations of a class of nuclear reactors with delayed neutrons", Arch. Ration. Mech. Anal. 31, 151-172, (1968).

[17] N. Levinson, "A nonlinear Volterra equation arising in the theory of superfluidity”, J. Math. Anal. Appl. 1, 1-11 (1960).

[18] W. Li, L. Panga, H. Sua, and K. Wang, "Global stability for discrete Cohen-Grossberg neural networks with finite and infinite delays", Appl. Math. Lett. 25, 2246-225 (2012).

[19] W.R. Mann, and F. Wolf, "Heat transfer between solids and gases under nonlinear boundary conditions", Quart. Appl. Math. 9, 163-184 (1951).

[20] P.H.A. Ngoc, and L.T. Hieu, "New criteria for exponential stability of nonlinear difference systems with time-varying delay", Internat. J. Control 86, No. 9, 1646-1651 (2013).

[21] P.H.A. Ngoc, T. Naito, J.S. Shin, and S. Murakami, "Stability and robust stability of positive linear Volterra difference equations", Internat. J. Robust Nonlinear Control 19, 552-568 (2008).

[22] Y.N. Raffoul, and Y.M. Dib, "Boundedness and stability in nonlinear discrete dystems with nonlinear perturbation", J. Difference Equ. Appl. 9, 853-862 (2003).

[23] Y. Song, and C.T.H. Baker, "Perturbation of Volterra difference equations", J. Difference Equ. Appl. 10, 379-397 (2004).

[24] T. Zhou, Y. Liu, X. Li, and Y. Liu, "A new criterion to global exponential periodicity for discrete-time BAM neural network with infinite delays", Chaos Solitons Fractals 39, 332-341 (2009). 